\title{
Pellino-1 promotes lung carcinogenesis via the stabilization of Slug and Snail through K63-mediated polyubiquitination
}

\author{
Yoon Kyung Jeon ${ }^{\star, 1,5}$, Chung Kwon Kim²,5, Kyung Rim Hwang ${ }^{2}$, Hye-Young Park ${ }^{2}$, Jaemoon Koh ${ }^{1,3}$, Doo Hyun Chung ${ }^{1,3}$, \\ Chang-Woo Lee ${ }^{2,4}$ and Geun-Hyoung Ha ${ }^{*, 2}$
}

Pellino-1 is an E3 ubiquitin ligase acting as a critical mediator for a variety of immune receptor signaling pathways, including Tolllike receptors, interleukin-1 receptor and T-cell receptors. We recently showed that the Pellino-1-transgenic (Tg) mice developed multiple tumors with different subtypes in hematolymphoid and solid organs. However, the molecular mechanism underlying the oncogenic role of Pellino-1 in solid tumors remains unknown. Pellino-1-Tg mice developed adenocarcinoma in the lungs, and Pellino-1 expression was higher in human lung adenocarcinoma cell lines compared with non-neoplastic bronchial epithelial cell lines. Pellino-1 overexpression increased the cell proliferation, survival, colony formation, invasion and migration of lung adenocarcinoma cells, whereas Pellino-1 knock-down showed the opposite effect. Pellino-1 overexpression activated PI3K/Akt and ERK signaling pathways and elicited an epithelial-mesenchymal transition (EMT) phenotype of lung adenocarcinoma cells. Pellino-1-mediated EMT was demonstrated through morphology, the upregulation of Vimentin, Slug and Snail expression and the downregulation of E-cadherin and $\beta$-catenin expression. Notably, Pellino-1 had a direct effect on the overexpression of Snail and Slug through Lys63-mediated polyubiquitination and the subsequent stabilization of these proteins. Pellino-1 expression level was significantly correlated with Snail and Slug expression in human lung adenocarcinoma tissues, and lung tumors from Pellino-1-Tg mice showed Snail and Slug overexpression. The Pellino-1-mediated increase in the migration of lung adenocarcinoma cells was mediated by Snail and Slug expression. Taken together, these results show that Pellino-1 contributes to lung tumorigenesis by inducing overexpression of Snail and Slug and promoting EMT. Pellino-1 might be a potential therapeutic target for lung cancer.

Cell Death and Differentiation (2017) 24, 469-480; doi:10.1038/cdd.2016.143; published online 23 December 2016

Epithelial-mesenchymal transition (EMT) has important roles in the formation of the body plan and in the differentiation of various tissues and organs. ${ }^{1,2}$ Moreover, EMT has a critical role in the carcinogenic process through various molecular pathways. ${ }^{1,3,4}$ EMT promotes tumor cell invasion and metastasis, leading to the generation of cancer cells with stem cell-like characteristics and resistance to chemotherapy., The loss of expression of the epithelial marker E-cadherin is a hallmark of EMT, associated with the upregulation of E-cadherin transcriptional repressors, such as Snail, Slug, Twist, ZEB1 and ZEB2.$^{6-9}$ In addition, EMT is regulated through various signaling networks including ERK, MAPK, PI3K/Akt, TGF- $\beta$, Wnt/ $\beta$-catenin and Notch pathways. ${ }^{2,4,10-12}$

Lung cancer remains the leading cause of cancer-related incidence and mortality worldwide. ${ }^{13,14}$ Previous studies have suggested a role for EMT in lung cancer, although the molecular mechanism driving EMT remains elusive. The loss of E-cadherin through genetic or epigenetic alterations is correlated with the aggressive behavior of lung cancer. ${ }^{4,15,16}$ Moreover, transcriptional repressors of E-cadherin are upregulated in lung cancer, associated with tumor progression and poor prognosis of patients. ${ }^{17-20}$

The Pellino family comprises three evolutionally conserved members (Pellino-1/2/3) that possess Ring-like motifs, a defining feature of the RING class of E3 ubiquitin ligases. ${ }^{21}$ Pellino proteins interact with various E2 enzymes to form Lys11-, Lys48- and Lys63-linked polyubiquitin chains in vitro and in vivo. ${ }^{22-24}$ Recent studies have demonstrated that Pellino-1 regulates innate and adaptive immune signaling pathways, including Toll-like receptors (TLRs), interleukin-1 receptor (IL-1R) and T-cell receptor signaling, ${ }^{25,26}$ and also involves in regulating the proliferation and activation of $\mathrm{B}$ and T cells. However, the biological role of Pellino in tumorigenesis remains unclear. Recent studies have indicated that Pellino-1 activates NF-KB and MAPK signaling pathways in human and murine immune cells. ${ }^{27-29}$ Interestingly, we previously used

\footnotetext{
${ }^{1}$ Department of Pathology, Seoul National University Hospital, Seoul National University College of Medicine, Seoul, Republic of Korea; ${ }^{2}$ Department of Molecular Cell Biology, Samsung Biomedical Research Institute, Sungkyunkwan University School of Medicine, Suwon, Republic of Korea; ${ }^{3}$ Department of Biomedical Sciences, Seoul National University College of Medicine, Seoul, Republic of Korea and ${ }^{4}$ Department of Health Sciences and Technology, Samsung Advanced Institute for Health Sciences and Technology, Sungkyunkwan University, Suwon, Republic of Korea

*Corresponding author: YK Jeon, Department of Pathology, Seoul National University Hospital, Seoul National University College of Medicine, 101 Daehak-ro, Jongno-gu, Seoul 03080, Republic of Korea. Tel: +82 22072 1347; Fax: +82 2743 5530; E-mail: junarplus@chol.com

or G-H Ha, Department of Molecular Cell Biology, Samsung Biomedical Research Institute, Sungkyunkwan University School of Medicine, 2066 Seobu-ro, Jangan-gu, Suwon-si 16419, Gyeonggi-do, Republic of Korea. Tel: +82 31 2996153; Fax: +82 31 2996109; E-mail: hasarang00@skku.edu

${ }^{5}$ Co-first authors.

Received 18.5.16; revised 26.10.16; accepted 08.11.16; Edited by J-C Marine; published online 23.12.2016
} 
transgenic $(\mathrm{Tg})$ mice to assess the gain of function for Pellino1 and observed the increased incidence of tumors in the lungs, livers, lymph nodes, and other organs of Pellino-1-Tg mice. ${ }^{30}$ This observation suggested that Pellino-1 might contribute to the tumor development of different types. ${ }^{30}$ Moreover, we provided the first evidence that Pellino- 1 is a novel oncogene contributing to B-cell lymphomagenesis through induction of BCL6 via Lys(K)63-mediated polyubiquitination and consequent stabilization. ${ }^{30}$ However, the mechanism by which Pellino-1 promotes development of epithelial tumor in lungs remains unknown. Thus, we addressed this issue here.

In this study, we demonstrated a novel mechanism through which Pellino-1 contributes to lung tumorigenesis by promoting EMT. The results showed that the Pellino-1-mediated ubiquitination and overexpression of Slug and Snail promotes EMT in lung cancer.

\section{Results}

Aberrant overexpression of Pellino-1 promotes lung cancer development. Multiple tumors were observed in the lungs of Pellino-1-Tg mice, but not in non-Tg littermates (Figure 1a). Pellino-1 expression was much higher in the lung tissues of Pellino-1-Tg mice compared with non-Tg mice (Figure 1a). Histopathological examination of the lungs of Pellino-1-Tg mice showed adenocarcinoma (F3-31), dysplasia (F2-35) and atypical adenomatous hyperplasia (AAH)-like lesions (F5-106) (Figure 1b).

In non-neoplastic human lungs, Pellino-1 was highly expressed in bronchial epithelial cells, moderately expressed in alveolar macrophages and weakly expressed in alveolar pneumocytes (Figure 1c, upper). Pellino-1 was variably expressed in human lung cancer tissues (Figure 1c, middle) with the highest frequency in adenocarcinoma (Figure 1c, lower) among non-small cell lung cancer (NSCLC). Pellino-1 expression was evaluated in an extended cohort of pulmonary adenocarcinoma $(n=491)$. Pellino-1 overexpression was significantly higher in patients with adenocarcinoma harboring EGFR mutation, increased EGFR gene copy numbers and MET expression (Supplementary Table S1). However, Pellino1 expression was not an independent prognostic factor in multivariate analysis for disease-free survival after surgical resection of the tumor (data not shown). Pellino-1 expression was observed up to $65 \%(11 / 17)$ of lung adenocarcinoma cell lines with a relative value of expression exceeding 1.5 compared with non-neoplastic lung cell lines (BEAS-2B and WI-26) (Figure 1d). Together, these data suggest that the overexpression of Pellino-1 might be involved in the development of lung adenocarcinoma.

Pellino-1 promotes proliferation, survival, migration, invasion and oncogenic transformation in lung cancer cells. To evaluate the functional significance of Pellino-1 in lung tumorigenesis, Pellino-1 in A549 cells was overexpressed or knock-downed by transfection with Myc-Pellino-1, shPellino-1 or 3'-untranslated region (UTR) shPellino-1 (Figure 2a). The overexpression of Pellino-1 in A549 cells increased cell proliferation, whereas the depletion of Pellino-1 reduced cell proliferation (Figure $2 b$ ). The overexpression of
Pellino-1 enhanced cell migration and invasion, whereas the depletion of Pellino-1 suppressed the migration and invasion of A549 cells (Figures 2c and d). Similar results were observed in Pellino-1-overexpressing or knocked-down H1299 cells as well as in Pellino-1-overexpressing BEAS-2B cells (Supplementary Figures S1 and S2). The overexpression of Pellino-1 promoted anchorage-independent growth and colony-forming ability, whereas the depletion of Pellino-1 resulted in the opposite effect in A549 cells (Figure 2e). Moreover, in in vivo xenograft model, tumor growth was significantly decreased in mice injected with Pellino-1 knocked-down A549 cells compared with control (Figures $2 f$ and g). In contrast, Pellino-2 and -3 ( $\alpha$ and $\beta$ ) had no influence on the cell proliferation, survival, migration and invasion in A549 cells (Supplementary Figures S3a-d). Together, these data suggest that Pellino-1 promotes the proliferation, migration, invasion, tumor growth and oncogenic transformation of lung cancer cells.

Pellino-1 promotes the EMT and activates PI3K/Akt and ERK signaling pathways in lung cancer cells. Of note, Pellino-1-overexpressing A549 and H1299 cells displayed stretched or elongated spindle cell morphology, whereas the depletion of Pellino-1 increased cell-to-cell contacts (Figure 3a). Thus, it was hypothesized that EMT might be involved in Pellino-1-mediated lung tumorigenesis. Consistent with morphological changes, the overexpression of Pellino-1 reduced the expression of epithelial markers, including $E$-cadherin and $\beta$-catenin, but increased the expression of mesenchymal markers, including Vimentin, Snail, and Slug, in A549 and H1299 cells (Figure 3b, left). No significant changes were observed in Twist and ZEB1 expression (data not shown). Moreover, the depletion of Pellino-1 reduced the expression of Vimentin, Snail and Slug but increased the E-cadherin and $\beta$-catenin expression (Figure $3 b$, right). The expression of E-cadherin is negatively regulated by transcription factor, such as Snail, Slug, Twist and ZEB1. ${ }^{31-33}$ Both protein and mRNA expression of Snail and Slug were markedly increased in Pellino-1-overexpressing cells (Figures $3 b$ and $c$ ) and decreased in Pellino1-depleted cells (Figures $3 b$ and $d$ ) compared with control cells. In contrast, E-cadherin expression was markedly decreased in Pellino-1-overexpressing cells (Figures $3 \mathrm{~b}$ and c) and increased in Pellino-1-depleted cells (Figures $3 \mathrm{~b}$ and d) at both protein and mRNA levels. These findings suggest that Pellino-1 has an important role in the progression of EMT through the upregulation of E-cadherin repressors, such as Snail and Slug.

Cell survival and EMT are associated with activation of PI3K/Akt and MAPK/ERK pathways. ${ }^{34}$ PI3K/Akt activation phosphorylates and inactivates GSK $3 \beta$ and thus inhibits active GSK3 $\beta$ (i.e., dephosphorylated form)-induced degradation of Snail protein. ${ }^{35,36}$ Thus, it was examined whether Pellino-1 regulates these signaling pathways. The phosphorylation of Akt, ERK1/2 and GSK3 $\beta$ was elevated in Pellino-1overexpressing A549 and H1299 cells (Figure 3e, left), but decreased in Pellino-1-depleted A549 and H1299 cells (Figure 3e, right). In Pellino-1-overexpressing A549 cells, LY294002 (PI3K inhibitor) and PD98059 (MEK inhibitor) reduced the Snail and Slug expression but increased the 


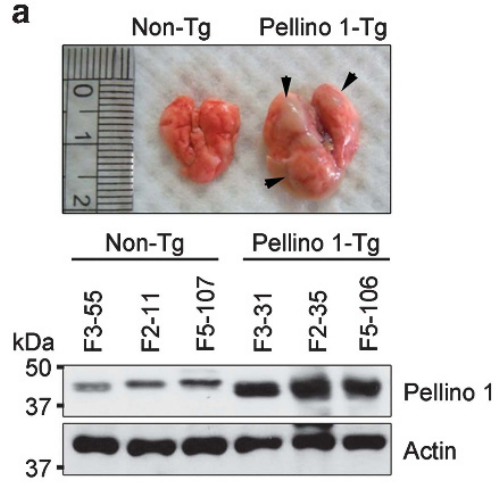

C
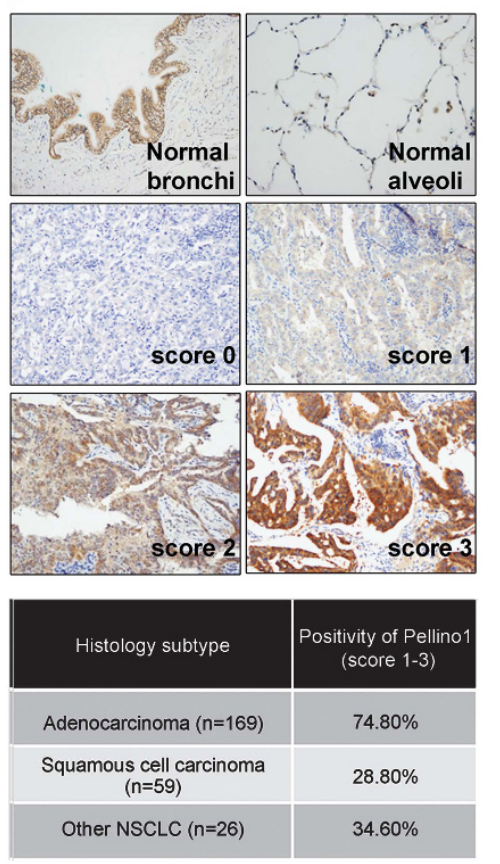
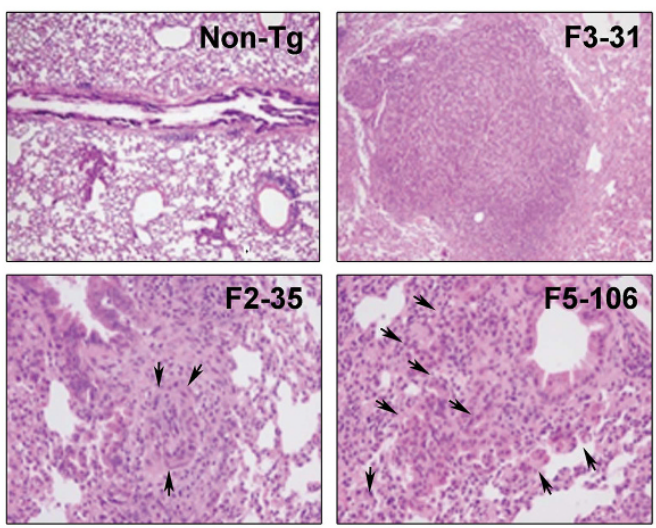

d
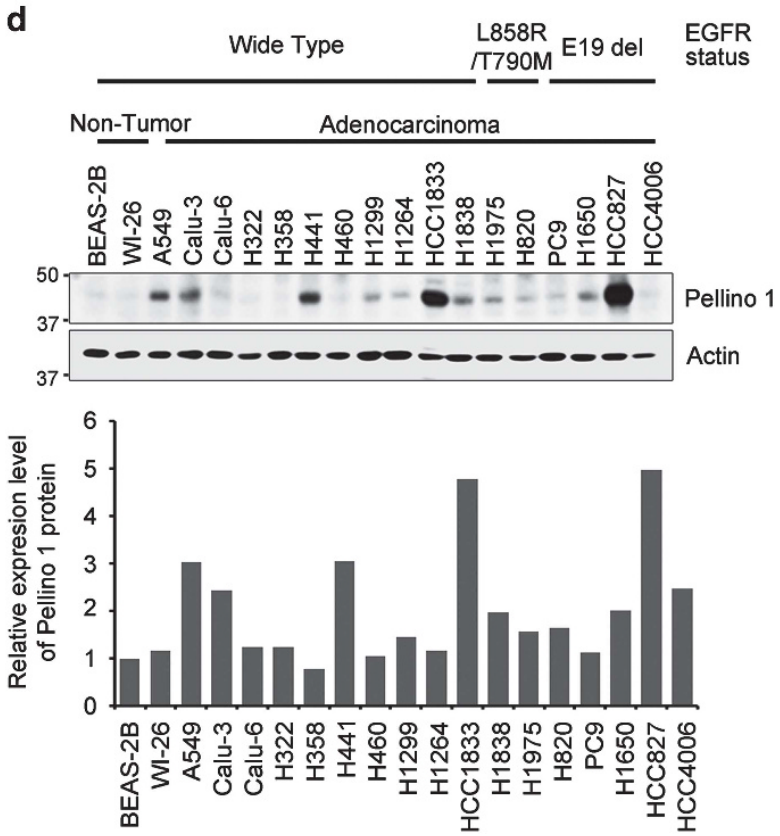

Figure 1 Pellino-1-Tg mice develop lung cancer and Pellino-1 is overexpressed in human lung cancers. (a) Representative macroscopic images showing lung tumors (arrows) in Pellino-1-Tg mice (top). Lysates of non-Tg and Pellino-1-Tg lung tissues were subjected to immunoblotting for Pellino-1 and actin (bottom). (b) Microscopic images for lung adenocarcinoma (F3-31), dysplasia (F2-35) and AAH-like lesions (F5-106) developed in Pellino-1-Tg mice. (c) Representative IHC images for Pellino-1 in normal human lung tissues (upper) and lung cancers (middle and lower) (original magnification, $\times 400$ ). Pellino-1 expression rate was analyzed according to the histological subtype of human lung cancer including adenocarcinoma, squamous cell carcinoma and other NSCLC histology (bottom table). (d) Two non-tumor human lung cell lines (BEAS-2B and WI-26) and 17 human lung cancer cell lines (all adenocarcinomas except for H460) with WT EGFR or mutated EGFR were analyzed for Pellino-1 expression using immunoblotting (top). Pellino-1 expression level was quantified through scanning densitometry with actin as an internal control (bottom)

E-cadherin expression (Figure 3f). Overexpression of GSK3 $\beta$ significantly reduced the levels of Slug and Snail as previously reported. ${ }^{35,37}$ Thus, it was determined whether overexpression of Pellino-1 enhances the stability of Snail and Slug proteins in GSK3 $\beta$-overexpressing cells. GSK3 $\beta$ overexpression in A549 cells reduced the Snail and Slug expression, whereas the overexpression of Pellino-1 in the GSK3 $\beta$ overexpressing cells significantly recovered the levels of Slug and Snail (Supplementary Figure S4). This data suggest that Pellino-1 might prevent the GSK3 $\beta$-mediated Slug and Snail degradation. Taken together, these results indicated that Pellino-1-mediated activation of PI3K/Akt and ERK signaling pathways might be implicated in Pellino-1-mediated cell proliferation and EMT progression.
Pellino-1 interacts and regulates Slug and Snail stabilization via K63-mediated ubiquitination. Pellino-1 is an E3 ligase. Thus, it was hypothesized that Pellino-1 might interact with Slug and Snail to regulate their stabilities. Extracts from asynchronously growing TAP and TAP-Pellino-1 (Flag-tagged Pellino-1) transfected 293T cells were subjected to pull-down assays (Figure 4a), which showed Pellino-1 interacts with endogenous Snail or Slug but not with E-cadherin and $\beta$-catenin. Next, GST-Pellino-1 fusion protein was incubated with cellular extracts from asynchronized A549 cells (Figure 4b), and GST-Slug or GST-Snail fusion proteins were incubated with purified His-tagged Pellino-1 (Figure 4c). Immunoprecipitation assay for Pellino-1 and Snail or Slug was also performed in 293T and A549 cells (Figure 4d). 


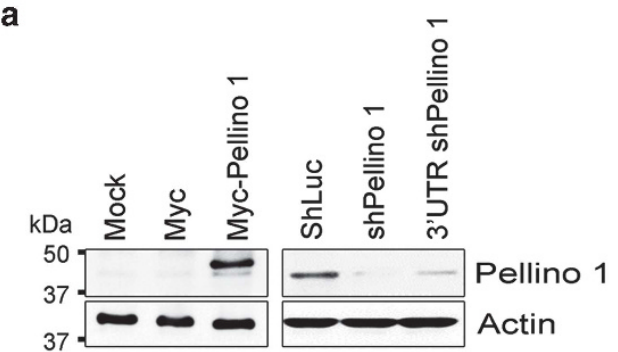

b

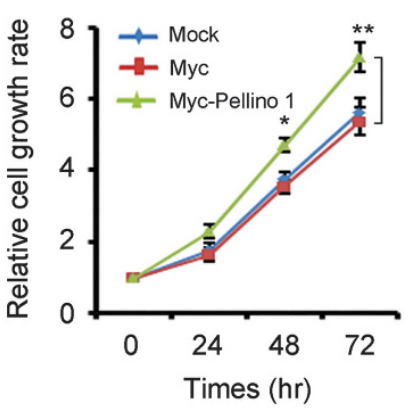

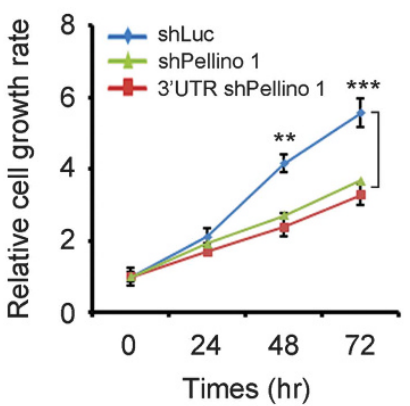

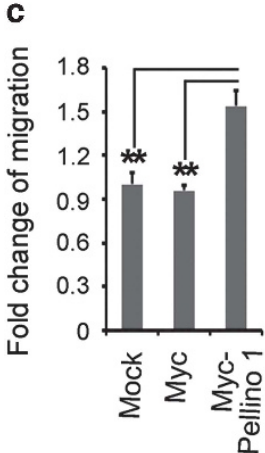

f

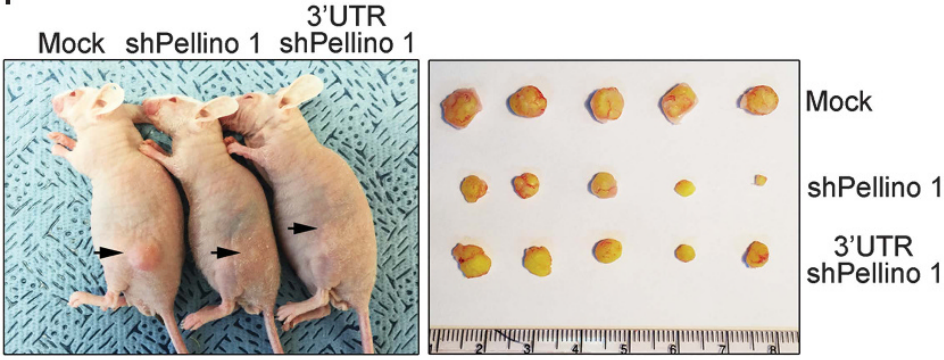

d

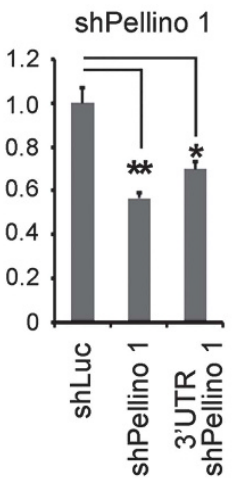

d

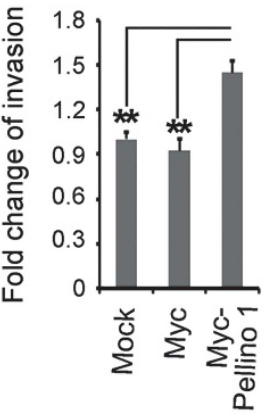

shPellino 1

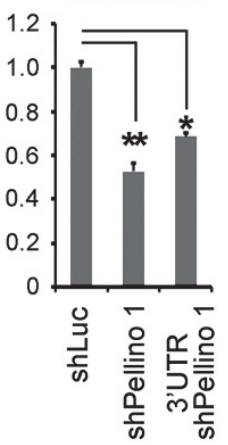

shPellino 1

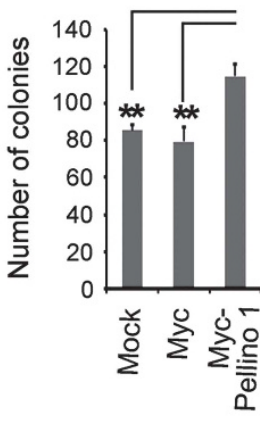

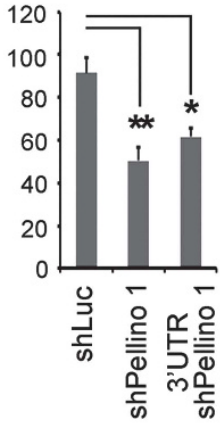

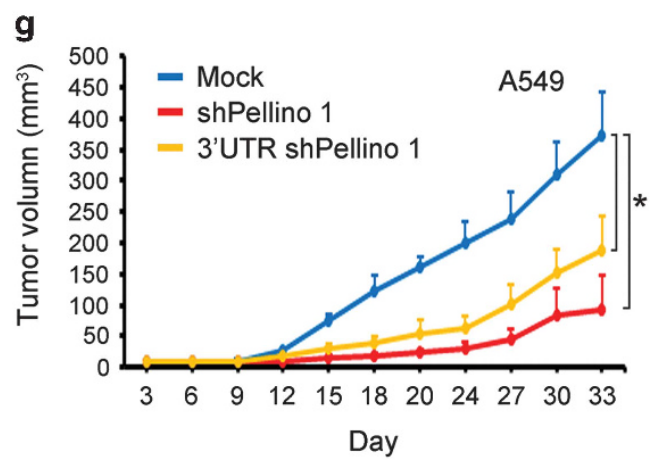

Figure 2 Pellino-1 overexpression enhances the cellular proliferation, migration, invasion and oncogenic transformation in A549 cells. (a) A549 cells were transfected with Myc (control), Myc-tagged Pellino-1, shLuc (control), Pellino-1-targeted shRNA (shPellino-1) or 3'-UTR Pellino-1-targeted shRNA (3'-UTR shPellino-1), and subjected to immunoblotting for Pellino-1 and actin. (b) MTT assay was performed to estimate the cell proliferation of A549 cells with Pellino-1 overexpression or knock-down. (c and d) A549 cells with Pellino-1 overexpression (Myc-Pellino-1) or Pellino-1 depletion (shPellino-1 and 3'-UTR shPellino-1) were pretreated with $10 \mu \mathrm{g} / \mathrm{ml}$ mitomycin C for $1 \mathrm{~h}$ at $37^{\circ} \mathrm{C}$, washed twice with PBS and then were subjected to transwell migration (c) and invasion assays (d). (e) A549 cells with Pellino-1 overexpression or Pellino-1 depletion were subjected to colony-forming assay. The numbers of colonies were counted in four randomly selected microscopic fields per plates. (f-g) A549 cells $\left(5 \times 10^{6}\right)$ transfected with shLuc, shPellino- 1 or 3'-UTR shPellino-1 were inoculated subcutaneously into the flank of athymic nude mice (five mice per group). Animals were monitored up to 33 days and tumor size was measured using an electronic caliper at 3-day intervals. All data are shown as the means \pm S.D. of at least three independent experiments. The $P$-values were calculated using unpaired Student's $t$-test. ${ }^{*} P<0.05 ;{ }^{* *} P<0.01 ;{ }^{* *} P<0.005$

Immunoprecipitation assay was also performed using GFP fusion Pellino-1 full-length (FL), ring-like domain deleted $(\triangle \mathrm{C})$ and ring-like domain only $(\mathrm{C})$ plasmids to see interactions with Snail and Slug (Supplementary Figure S5). Results from these assays suggested that Pellino-1 interacts with Snail and Slug via FHA domain of Pellino-1.

Of note, the overexpression of FL Pellino-1 (Pellino-1-FL) increased the expression of Slug and Snail in A549 cells, but the overexpression of Pellino-1 C-terminal RING domain deletion mutant (Pellino-1- $\Delta \mathrm{C}$ ), which has defect in E3 ligase activity, did not (Supplementary Figures S6a and b). The Akt and ERK1/2 phosphorylation and cell migration was increased by Pellino-1-FL in A549 cells, but not by Pellino-1- $\Delta \mathrm{C}$ (Supplementary Figures S6b-c). These data suggested that Pellino-1's function in A549 cells might depend on its E3 ligase activity. Thus, ubiquitination status of Slug and Snail affected by Pellino-1 was evaluated. To this end, HCT116 cells were transfected with Myc-Pellino-1-FL or Myc-Pellino-1- $\Delta \mathrm{C}$ in combination with HA-Ub and Flag-Slug or Flag-Snail, and subjected to immunoprecipitation with an anti-Flag antibody and subsequent immunoblotting. Interestingly, the polyubiquitinated forms of Slug (Figure 4e, left) and Snail (Figure 4e, right) were evident in cells transfected with Pellino-1-FL, but not in cells transfected with Pellino-1- $\Delta$ C. To further examine 
a
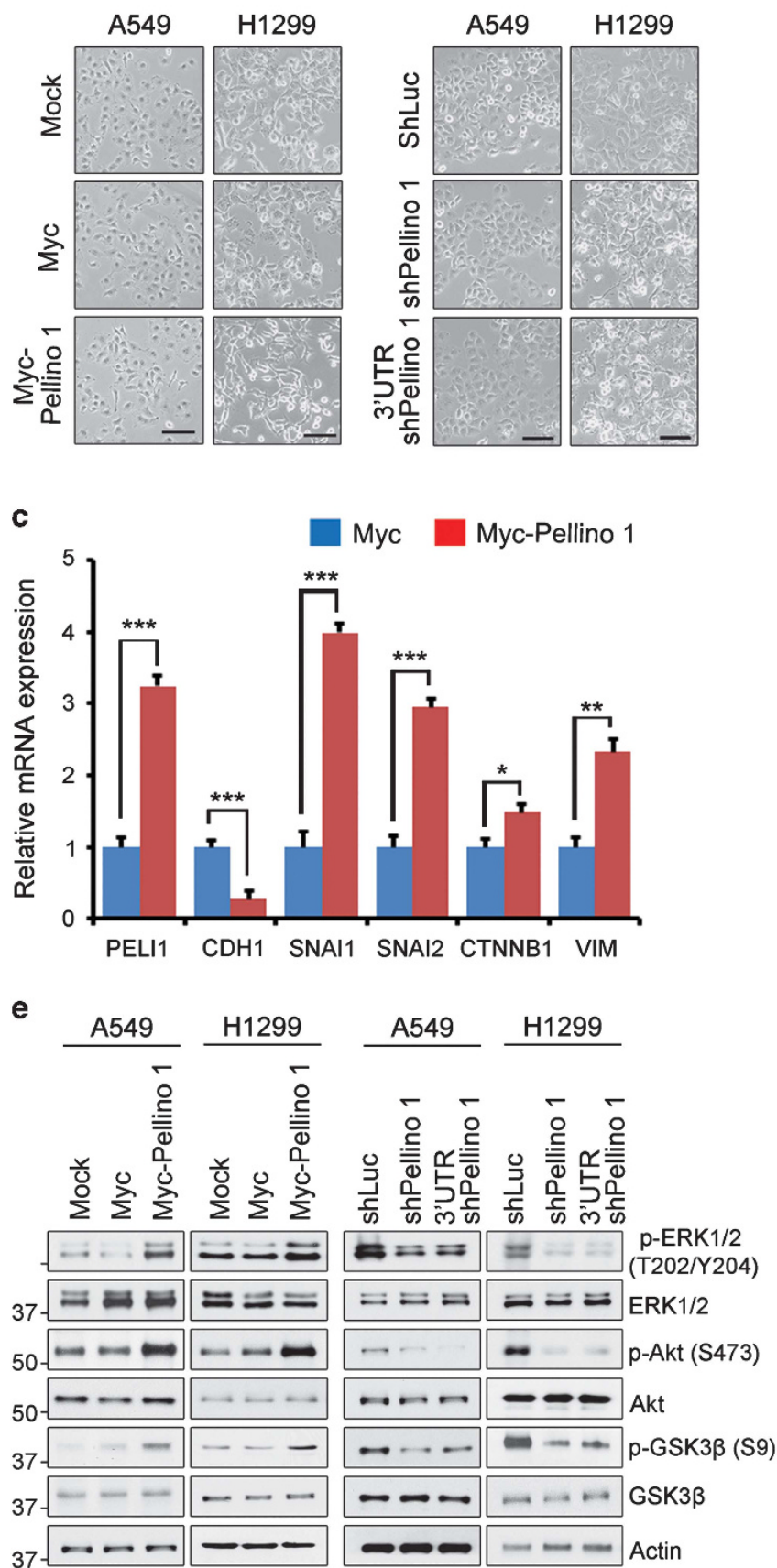

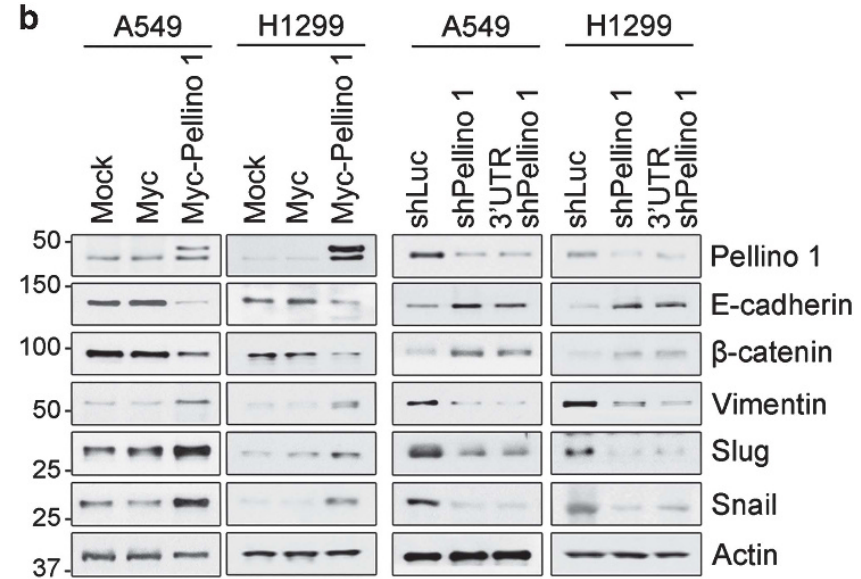

d

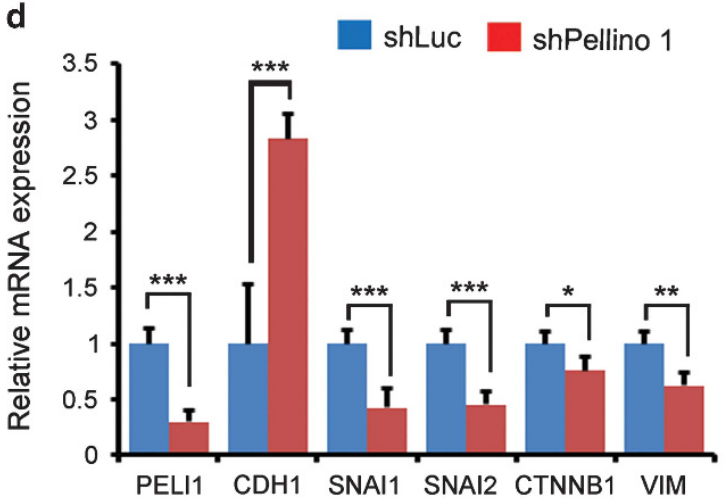

f

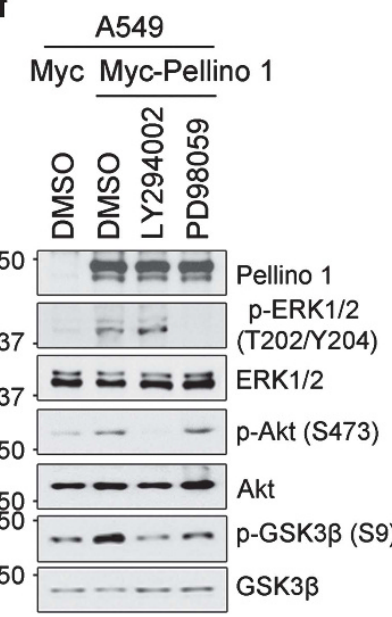

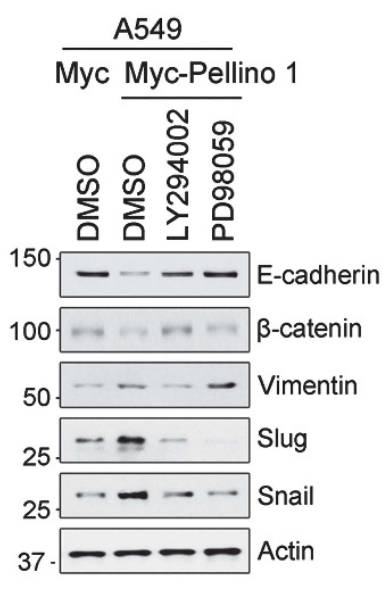

Figure 3 Pellino-1 promotes the EMT phenotype and activates the PI3K/Akt and ERK signaling pathways. (a) Representative phase-contrast images for Pellino-1overexpressing A549 and H1299 cells (left) and Pellino-1-depleted A549 and H1299 (right). The scale bars represent $100 \mu \mathrm{m}$. (b) A549 and H1299 cells were transfected with the Myc or Myc-Pellino-1 (left), and the shLuc, shPellino-1 or 3'-UTR shPellino-1 (right), and subjected to immunoblotting for Pellino-1, E-cadherin, $\beta$-catenin, Vimentin, Slug, Snail and actin. (c and d) Analysis of PELI1 (encoding to Pellino-1), CDH1 (encoding to E-cadherin), SNAl1 (encoding to Snail), SNAI2 (encoding to Slug), CTNNB1 (encoding to $\beta$-catenin), VIM (encoding to Vimentin) mRNA levels was performed by qRT-PCR in Pellino-1-overexpressing (c) and -knocked-down (d) A549 cells. (e) A549 and H1299 cells were transfected with the Myc or Myc-Pellino-1 (left), and the shLuc, shPellino-1 or 3'-UTR shPellino-1 (right), and subjected to immunoblotting for p-ERK1/2 (T202/Y204), ERK1/2, p-Akt (S473), Akt, p-GSK3 $\beta$ (S9), GSK3 $\beta$ and actin. (f) A549 cells were transfected with the Myc or Myc-Pellino-1, and Pellino-1-overexpressing A549 cells were subsequently treated with LY294002 $(20 \mu \mathrm{M})$ or PD98059 $(20 \mu \mathrm{M})$. After incubation of $12 \mathrm{~h}$, the cells were lysed and subjected to immunoblotting with the indicated antibodies. All data are shown as the means \pm S.D. of at least three independent experiments. The $P$-values were calculated using unpaired Student's $t$-test. ${ }^{*} P<0.05$; ${ }^{\star *} P<0.01 ;{ }^{* \star *} P<0.005$ 

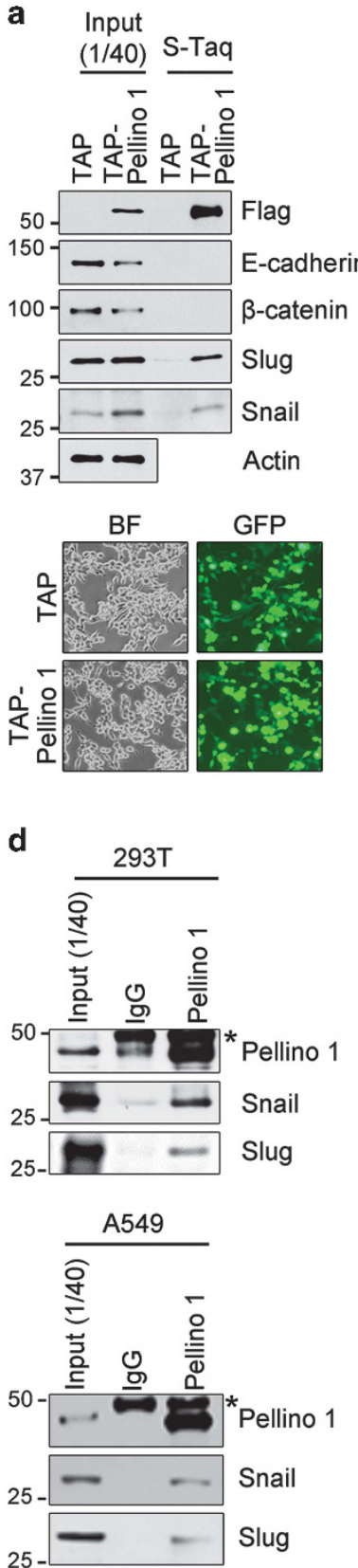

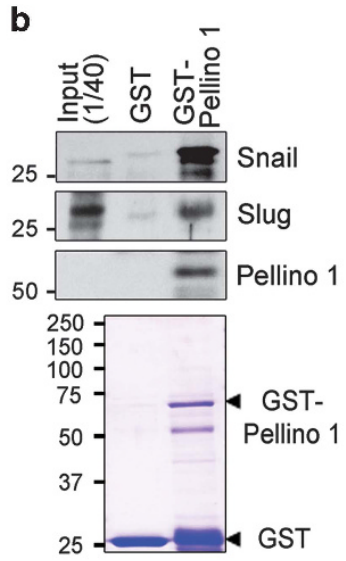

C
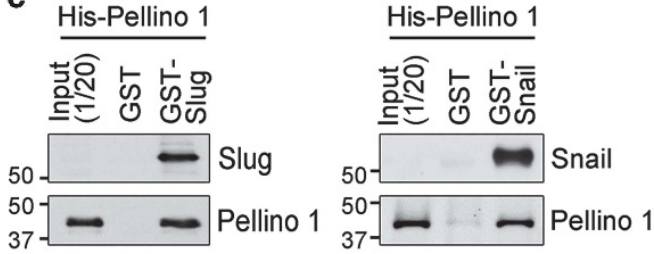

e
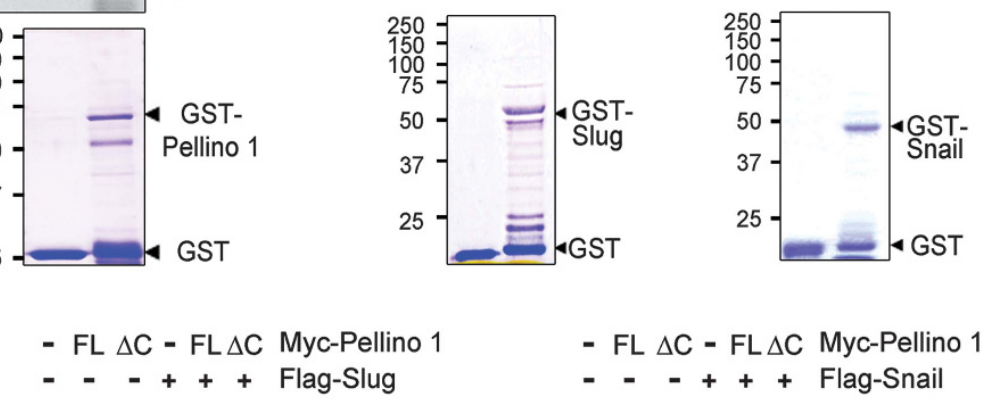

+++++ HA-Ub
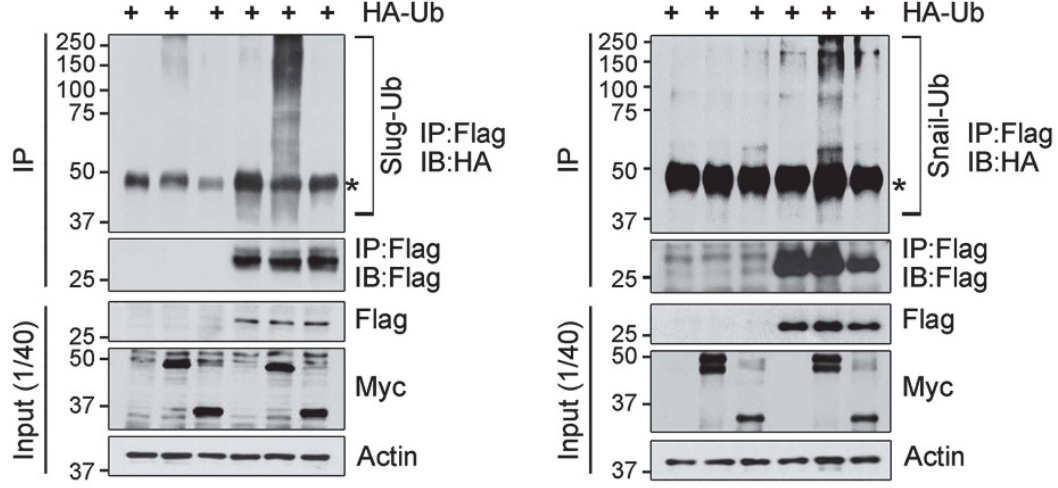

$\mathbf{f}$

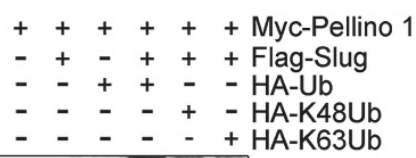
++++++ Myc-Pellino 1
-+-+++ Flag-Snail
- + + - - HA-Ub
- - + + HA-K48Ub
$-{ }_{-}-{ }_{-}+\mathrm{HA}-\mathrm{K}$
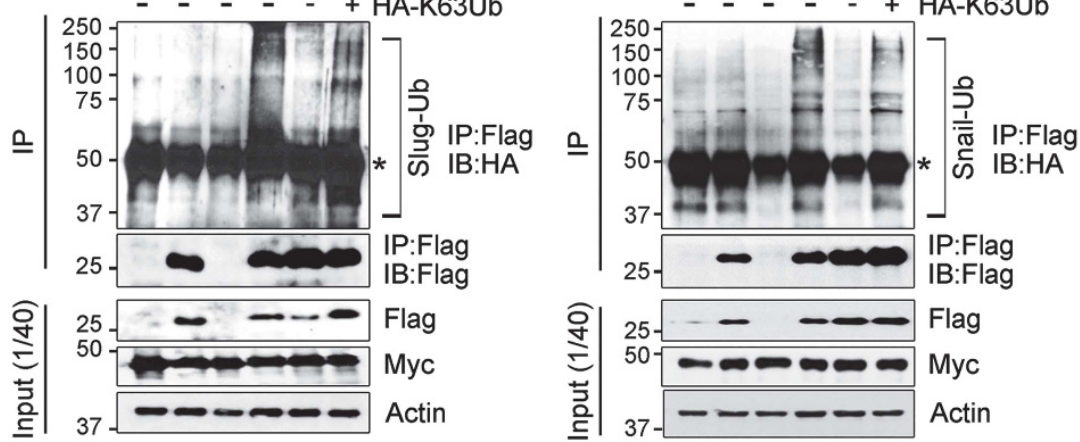

Figure 4 Pellino-1 directly interacts with Slug and Snail, and promotes K63-mediated polyubiquitination of Slug and Snail. (a) 293T cells were transfected with TAP (control), TAP-Pellino-1 (Flag-tagging Pellino-1). At $36 \mathrm{~h}$ post-transfection, the cells were inspected for GFP expression under microscope (lower) and harvested and isolated through tandem affinity purification (TAP). The bound proteins were immunoblotted with the indicated antibodies (upper). Transfection efficiency was evaluated by inspecting cells expressing GFP under microscope (BF, bight filed; lower). (b) A549 cell lysates were incubated with GST or GST-Pellino-1, and subjected to immunoblotting with the indicated antibodies. (c) GST, GST-Slug or GST-Snail fusion proteins were incubated with purified His-Pellino-1, and subjected to immunoblotting for Slug, Snail and Pellino-1. (d) 293T (upper panel) or A549 (bottom panel) cell lysates were immunoprecipitated with anti-Pellino-1 antibody and immunoblotted with anti-Snail, anti-Slug and anti-Pellino-1 antibodies. Asterisk denotes nonspecific band. (e) HCT116 cells were transfected with Myc-Pellino-1 FL or Myc-Pellino-1- $\Delta \mathrm{C}$ (ring domain deletion mutant) in combination with HA-Ub and Flag-Slug (left) or Flag-Snail (right). At $36 \mathrm{~h}$ post-transfection, the cells were harvested and immunoprecipitated with an anti-Flag antibody. The Slug or Snail protein complexes were subjected to immunoblotting with an anti-HA and anti-Flag antibodies. (f) HCT116 cells were transfected with Myc-Pellino-1, HA-UB, HA-UB K48, HA-Ub K63 and Flag-Slug (left) or Flag-Snail (right) in combination. At $36 \mathrm{~h}$ post-transfection, the cells were harvested and immunoprecipitated with an anti-Flag antibody. The Slug or Snail protein complexes were subjected to immunoblotting with anti-HA and anti-Flag antibodies 
the ubiquitination site of Slug and Snail through Pellino-1, HCT116 cells were transfected with Myc-Pellino- 1 in combination with HA-Ub, HA-K48Ub, HA-K63Ub, Flag-Slug or FlagSnail, and analyzed as above (Figure 4f). The overexpression of Pellino-1 increased the amount of HA-Ub and HA-Ub K63 polyubiquitinated forms of Slug and Snail, but not the HA-Ub K48 polyubiquitinated forms. In addition, endogenous polyubiquitinated forms of Snail and Slug proteins was evaluated in A549 cells transfected with TAP and TAPPellino-1 combined with His-Ub (Supplementary Figure S7). Overexpression of Pellino-1 (TAP-Pellino-1) increased the endogenous polyubiquitinated forms of Snail and Slug proteins compared with control cells (TAP) (Supplementary Figure S7). Together, these results indicate that Pellino-1 might directly regulate Slug and Snail via Lys(K)63-mediated polyubiquitination.

Pellino-1 expression promotes Slug and Snail stabilization in lung cancer cells. The relationship between Pellino-1, Slug and Snail in lung cancer cells was further evaluated using human lung non-neoplastic and neoplastic cell lines. Pellino-1 ${ }^{\text {Low }}$ BEAS-2B, H358 and PC9 cells were transfected with the Myc-Pellino-1, which resulted in an increase of Slug and Snail expression (Figure 5a). In contrast, Pellino- ${ }^{\text {High }} \mathrm{H} 441, \mathrm{H} 1838, \mathrm{H} 1975$ and HCC827 cells transfected with shPellino-1 or 3'-UTR shPellino-1 showed reduction of Slug and Snail expression (Figure 5b). Moreover, ectopic expression of Pellino-1 (WT) in A549 cells increased the expression of Snail and Slug in a dose-dependent manner (Figures 5c and d). However, ectopic expression of Pellino-1 $(\triangle \mathrm{C})$ in A549 cells had no effect on the expression of Snail and Slug (Figures $5 e$ and f). These result indicated that Pellino-1 partially affects the transcriptional levels of Snail and Slug.

Next, treatment with a proteasome inhibitor (MG132) increased the expression of Slug and Snail in both shLuc and a shPellino-1-transfected A549 cell, suggesting that Slug and Snail protein stability might be regulated via a proteasome pathway (Figure $5 \mathrm{~g}$ ). In addition, we investigated whether overexpression of Pellino-1 in Pellino-1-depleted A549 cells rescue the expression of Snail and Slug proteins. Interestingly, overexpression of Pellino-1 wild-type (WT) recovered the levels of Slug and Snail proteins in Pellino-1-depleted A549 cells, but Pellino-1 mutants ( $\triangle \mathrm{C}$ and $\mathrm{C}$ domain) did not (Figure 5h). Taken together, these pull-down and immunoprecipitation assays revealed that Pellino-1 directly interacts and regulates Slug and Snail stabilization via FHA domain and E3 ligase activity.

Consistently, the degradation of Slug and Snail was delayed and their expression levels maintained in relatively steadystate levels in Pellino-1-overexpressing A549 cells treated with cycloheximide (inhibitor of protein synthesis), whereas Slug and Snail expression gradually decreased in control cells (Figure 5i). Taken together with aforementioned ubiquitination assay data, these results indicate that Pellino-1 stabilizes Snail and Slug protein via K63-mediated polyubiquitination.

Lung tissue from Pellino-1-Tg mice developing adenocarcinoma or related lesions showed Slug and Snail overexpression compared with lung tissue from non-Tg mice (Supplementary Figure S8), suggesting that Pellino-1 might affect the stability of Slug and Snail proteins in vivo.
Slug and Snail are required for Pellino-1-mediated EMT and increased migration in lung cancer cells. To determine whether the expression of Slug and Snail was prerequisite for Pellino-1-mediated EMT phenotype, A549 cells were transfected with shSlug (shSlug\#1 and shSlug\#2) and shSnail (shSnail\#1 and shSnail\#2) (Supplementary Figure S9). The depletion of Slug and Snail upregulated E-cadherin expression in Pellino-1-overexpressing A549 cells (Figures $6 a$ and b). In addition, Slug and Snail knock-down counteracted the increased migration observed in Pellino-1overexpressing A549 cells (Figures $6 c$ and d). These data indicated that Slug and Snail might have an important role in the Pellino-1-mediated EMT and increase in the migration of lung cancer cells.

Correlation between Pellino-1, Slug and Snail expression levels in human lung adenocarcinoma tissues. Finally, the relationship between Pellino-1 and Slug or Snail expression was assessed in patients with lung adenocarcinoma. Representative immunohistochemistry (IHC) images for Pellino-1, Slug and Snail from patients who showed strong expression for both Pellino-1 and Slug or Snail, or those with little expression for both proteins are shown in Figures $6 e$ and g. Pellino-1 expression (i.e., score 1-3) and strong Slug expression (i.e., score 3 ) showed statistically significant positive correlation ( $P=0.016$ by Pearson $x^{2}$ test; Figure $6 \mathrm{f}$ ). A strong positive correlation between Pellino-1 and Snail IHC scores was observed (Spearman's $R h o=0.445, P=0.000$; Figure 6h). Moreover, patients with Pellino-1 expression showed significantly higher Snail expression (score 2, 3) ( $P=0.000$ by Pearson $x^{2}$ test; Figure 6i). Significant positive correlations between Pellino-1 and Slug or Snail expression were consistently observed in lung adenocarcinoma when separately analyzed according to the EGFR mutation status (Supplementary Figure S10). These results showed that Pellino-1 expression is positively correlated with Snail and Slug expression, particularly having a strong relationship with Snail, in human lung adenocarcinomas.

\section{Discussion}

We observed that the overexpression of Pellino-1 promotes the development of a variety of lymphoid and solid tumors in Pellino-1-Tg mice. ${ }^{30}$ Thus, the deregulation of Pellino-1 is thought to have an oncogenic role in human cancer. However, the molecular mechanisms underlying the functional relevance of Pellino-1 in solid tumors, including lung cancer, has never been addressed. In this study, through in vitro and in vivo models using multiple lung cancer cell lines and xenograft and validation with human lung cancer tissues, we demonstrated that Pellino-1 has an oncogenic role in lung cancer through the stabilization of Snail and Slug via K63-mediated polyubiquitination, thereby promoting the EMT phenomenon. Pellino-1 was also revealed to promote cell proliferation and oncogenic transformation and activate Akt and ERK in lung cancer cells.

Various growth factors, such as epidermal growth factor (EGF), TGF- $\beta$ and insulin-like growth factor- 1 induced EMT in lung cancer and EMT was correlated with metastases and invasiveness of lung cancer. ${ }^{17,38-42}$ This study provided a 
novel mechanism by which EMT is regulated in lung cancer. Pellino-1 was found to enhance cell proliferation and cell invasion and migration with the induction of EMT, as evidenced through cell morphology and the expression pattern of EMT-related markers. Pellino-1 induced EMT with the upregulation of Snail and Slug and downregulation of E-cadherin. The Snail and Slug, has a critical role in EMT, encoding transcriptional repressors in the E-cadherin promoter. $^{32,33,43}$ Through a series of biochemical analysis, we here demonstrated that Pellino-1 directly interacts with Slug and Snail and increases the stability of these proteins via K63mediated polyubiquitination, whereas Pellino-1 indirectly regulated E-cadherin.

Pellino-1 is an E3 ubiquitin ligase mediating K48- and K63linked polyubiquitination, which destines the fate of target proteins into proteosomal degradation versus stabilization. ${ }^{24,44}$ Moreover, recognition of substrate by Pellino protein is mediated by its forkhead-associated (FHA) domain, which

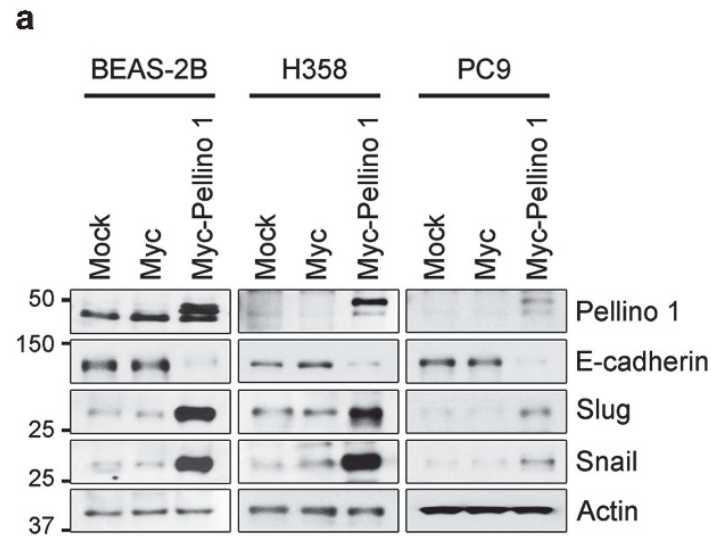

C

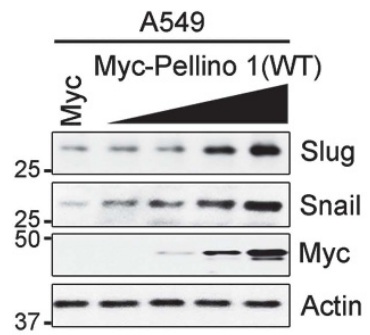

g

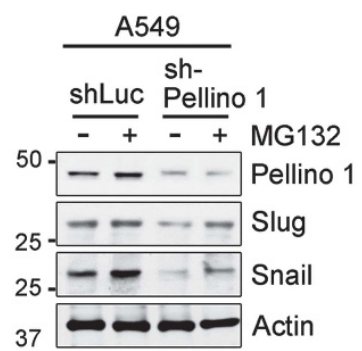

d

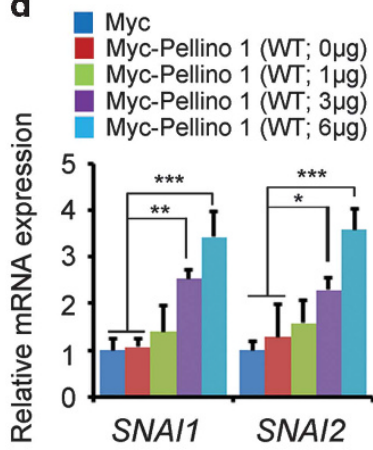

h

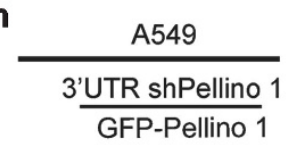

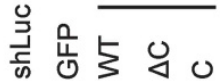

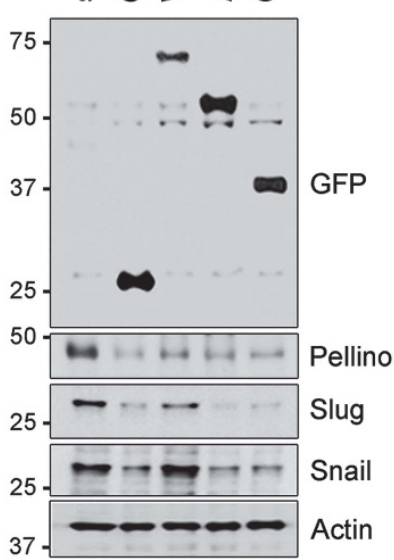

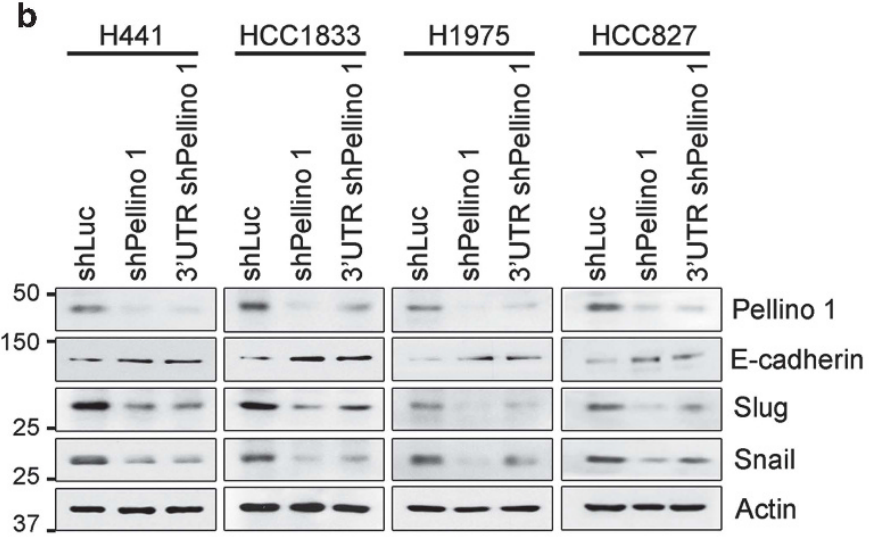

e

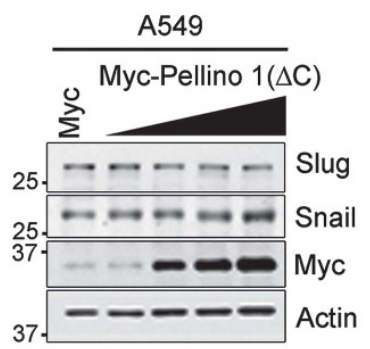

f

I Myc

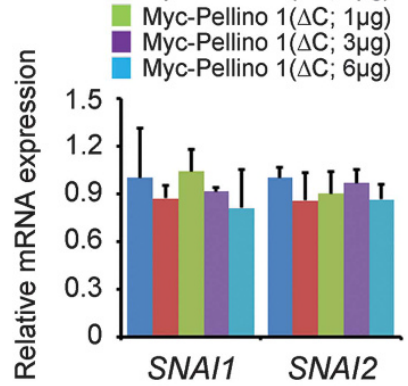

i

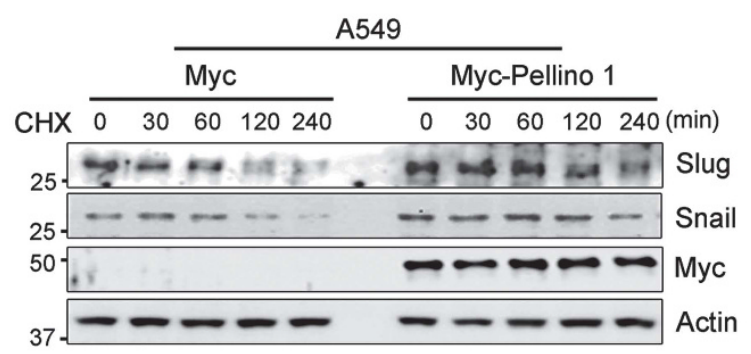

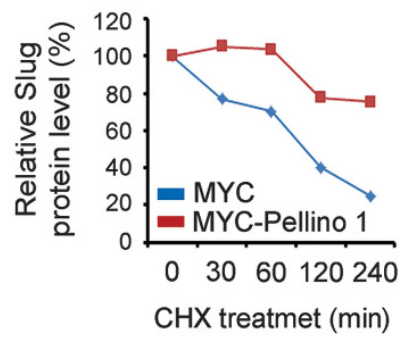


binds to specific phosphothreonine motifs (i.e., pTxxD pTxxl/L pTxxS/A pTxxy/M), thereby endowing unique substrate specificities to Pellino proteins. ${ }^{45}$ Until now, a few numbers of Pellino's substrates with FHA-binding motif have been identified, including IL-1 receptor-associated kinase-1 (IRAK1), receptor-interacting protein-1 (RIP1), TNF receptorassociated Factor-6 (TRAF6), RIP2, cRel and BCL6. ${ }^{30,44,45}$ The presence of potential FHA-binding phosphothreonine motifs was found in Snail and Slug protein (Supplementary Table S2), which further support Snail and Slug as novel substrate of Pellino-1.

Snail and Slug overexpression was associated with aggressiveness, chemotherapy resistance and poor survival in patients with lung cancer. ${ }^{2,17,46}$ This study showed that the overexpression of Pellino-1 is higher in lung adenocarcinoma rather than squamous cell carcinoma and other NSCLC histology. Moreover, Pellino-1 expression has a strong positive association with Snail or Slug expression in human lung adenocarcinoma. Together, these results indicate that Pellino-1 might function as an oncogene promoting EMT progress in human lung cancer, particularly in adenocarcinoma.

Pellino- 1 is known as a critical molecule cascading IL-1R or TLR signaling and activating NF-kB and MAPK pathways during inflammatory and innate immune responses by mediating ubiquitination of signaling molecules. ${ }^{27,28,29}$ EGF promotes EMT through the upregulation of Snail and Slug and activates PI3K/Akt and ERK signaling pathways in lung cancer. ${ }^{10,17,47}$ Akt is a key regulator of NF-KB activation, and although initially implicated as distinct signaling pathways, the NF-kB and Akt signaling pathways often overlap. ${ }^{48,49}$ Constitutive NF-kB activation was involved in EMT through the upregulation of EMT-inducing transcription factors in lung cancer cells. ${ }^{50}$ Lung cancer cells overexpressing activated mutants Akt underwent EMT along with Snail and Slug activation. ${ }^{2,12,51}$ ERK signaling also upregulated the expression of Snail and Slug in lung cancer. ${ }^{17,52}$ In this study, Pellino1 activated PI3K/Akt and ERK signaling, which was involved in upregulation of Slug and Snail and the downregulation of E-cadherin in A549 cells. These results suggested that Pellino-1 might promote EMT of lung cancer cells indirectly through PI3K/Akt and ERK activation as well as directly through the Slug and Snail stabilization. Moreover, Pellino-1 increased the phosphorylation (inactive form) of GSK3 $\beta$ in lung cancer cells. Given that active GSK3 $\beta$ promoted the degradation of Slug and Snail, ${ }^{35,36}$ this study suggests that PIJK/Akt/GSK3 $\beta$ axis might also be involved in Pellino-1- mediated stabilization of Slug and Snail protein. However, the mechanism by which Pellino-1 activates PI3K/Akt and ERK pathway in lung cancer cells remains to be elucidated.

Meanwhile, the regulatory mechanism of Pellino-1 in lung cancer also remains elusive. Pellino-1 expression was higher in human pulmonary adenocarcinomas with EGFR mutation or increased gene copy number, and MET expression (Supplementary Table S1). However, there was no significant difference in the level of Pellino-1 between EGFR mutant and EGFR WT lung cancer cell lines (Figure 1d). EGF stimulation upregulated Pellino-1 expression in A549 cells, whereas EGFR inhibitor treatment decreased the Pellino-1 expression (Supplementary Figure S11). EGF-induced increase in cell migration and invasion were partly mediated by Pellino-1 (Supplementary Figure S11). Taken together, our data suggest that EGFR signaling might be involved in Pellino-1 upregulation in lung cancer but there is a possibility that another mechanisms are also involved. Thus, more studies are needed to clarify how Pellino-1 expression is regulated in lung cancer.

In summary, we provided the first evidence of a novel mechanism through which Pellino-1 contributes to lung tumorigenesis by promoting EMT through Snail and Slug K63-polyubiquitination and subsequent stabilization. These findings provide new insights into the role of Pellino-1 in carcinogenesis. Based on these results, we propose targeting the Pellino-1/Snail/Slug pathway as a potential therapeutic strategy to control lung cancer progression. Pellino-1 could be exploited for the development of effective therapeutic strategies for lung cancer.

\section{Materials and Methods}

Cell culture. Seventeen human lung cancer cell lines (A549, Calu-3, Calu-6, H322, H358, H441, H460, H1299, H1264, HCC1833, H1838, H1975, H820, PC9, H1650, HCC827, HCC4006; all adenocarcinoma except for H460, large cell carcinoma), non-neoplastic bronchial epithelial cell lines (BEAS-2B), lung fibroblastic cell lines (WI-26) and 293T cells were purchased from the American Type Culture Collection (Manassas, VA, USA). HCT116 cells were kindly gifted from Bert Vogelstein (Johns Hopkins Kimmel Cancer Center, Baltimore, MD, USA). BEAS-2B cells were maintained in bronchial epithelial cell growth medium (Lonza, Walkersville, MD, USA) and 10\% FBS (Hyclone, Logan, UT, USA) supplemented with antibiotics. Other cell lines were grown in 90\% RPMI-1640, DMEM or McCoy's medium and $10 \%$ FBS supplemented with antibiotics in a humidified $5 \% \mathrm{CO}_{2}$ atmosphere.

Plasmid construction and transfection. The FL CDNA sequence of the human Pellino-1 was PCR amplified using oligodT primers. Pellino-1- $\Delta C$ included $280 \mathrm{~N}$-terminal amino acids and lacked the C-terminal RING domain. Pellino-1 FL, Pellino-1- $\Delta C$ and Pellino-1-C were subcloned into Myc-, GST-, GFP- or His6-tagged

Figure 5 Pellino-1 promotes Slug and Snail stabilization in lung cancer cells. (a) BEAS-2B, H358 and PC9 cells (Pellino-1 ${ }^{\text {Low }}$ ) were transfected with Myc or Myc-Pellino-1. At $48 \mathrm{~h}$ post-transfection, the cells were harvested and subjected to immunoblotting for Pellino-1, E-cadhrin, Snail, Slug and actin. (b) H441, H1833, H1975 and HCC827 cells (Pellino-1 ${ }^{\text {High }}$ ) were transfected with shLuc, shPellino-1 or 3'-UTR shPellino-1. At $48 \mathrm{~h}$ post-transfection, the cells were harvested and subjected to immunoblotting for Pellino-1, E-cadhrin, Snail, Slug and actin. (c) A549 cells were transfected with Myc or Myc-Pellino-1 (WT; 0, 1, 3, $6 \mu$ g of plasmid). At 48 h post-transfection, the cells were harvested and subjected to immunoblotting for Myc, Snail, Slug and actin and (d) qRT-PCR analysis for SNAl1 (encoding to Snail) and SNAl2 (encoding to Slug). (e) A549 cells were transfected with Myc or Myc-Pellino-1 ( $\Delta \mathrm{C} ; 0,1,3,6 \mu \mathrm{g}$ of plasmid). At $48 \mathrm{~h}$ post-transfection, the cells were harvested and subjected to immunoblotting for Myc, Snail, Slug and actin and (f) qRT-PCR analysis for SNAl1 and SNAI2. (g) A549 cells were transfected with shLuc or shPellino-1 and subsequently treated with the proteasome inhibitor, MG132 (10 $\mu$ M) for $6 \mathrm{~h}$. The cells were subsequently subjected to immunoblotting for Slug, Snail, Pellino-1 and actin. (h) A549 cells were transfected with GFP, GFP-Pellino-1 (FL, ring-like domain deletion $(\Delta \mathrm{C})$, ring-like domain $(\mathrm{C})$ ), shLuc, or 3'-UTR shPellino-1 plasmids. At $36 \mathrm{~h}$ post-transfection, the cells were harvested and immunoprecipitated with indicated antibodies. (i) A549 cells were transfected with Myc or Myc-Pellino-1. At $36 \mathrm{~h}$ post-transfection, the cells were treated with cycloheximide (CHX) for indicated times. The cell lysates obtained at each time point were subjected to immunoblotting for Snail, Slug, Myc and actin (top). The results were quantified through scanning densitometry of the Slug or Snail protein level with actin as an internal control (bottom). All data are shown as the means \pm S.D. of at least three independent experiments. The $P$-values were calculated using unpaired Student's $t$-test. ${ }^{*} P<0.05 ;{ }^{* *} P<0.01 ;{ }^{* * *} P<0.005$ 


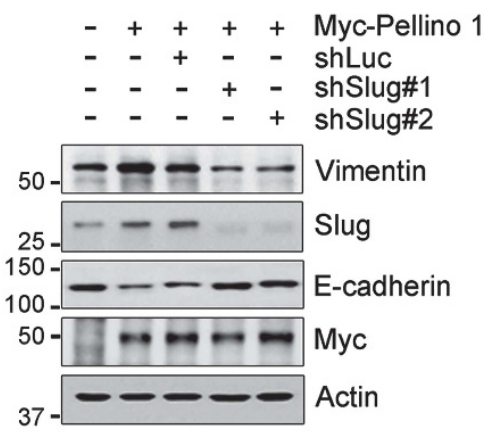

b

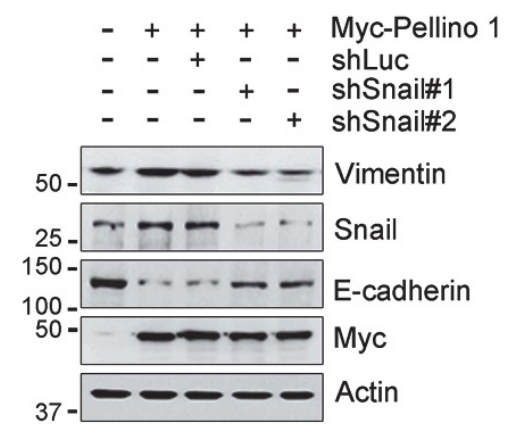

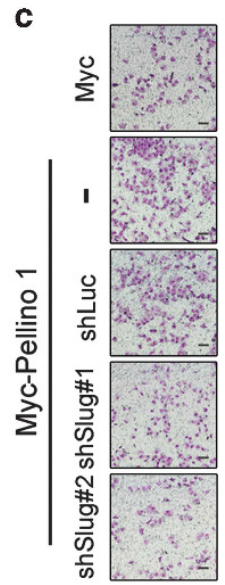

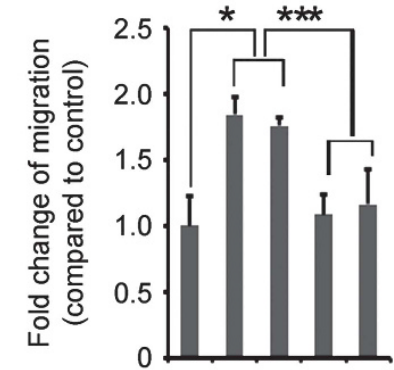

Myc-Pellino $1-++++$

shLuc $\quad-\quad+-$ shSlug\#1 - -++shSlug\#2 - $\quad-\quad-+$
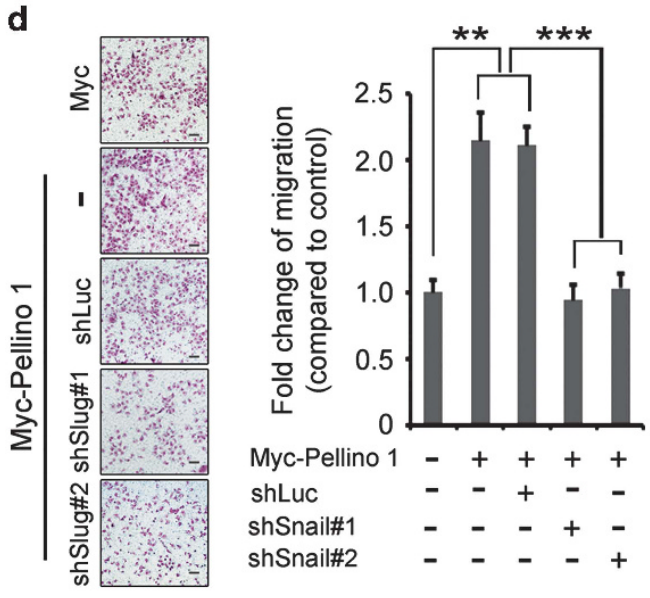

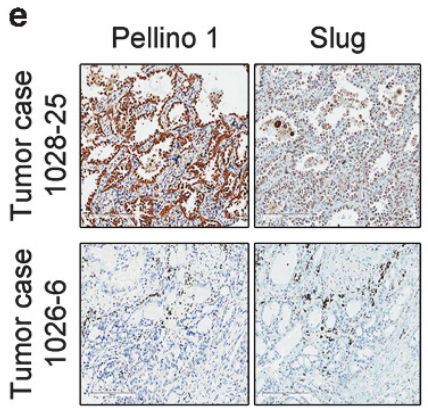

f

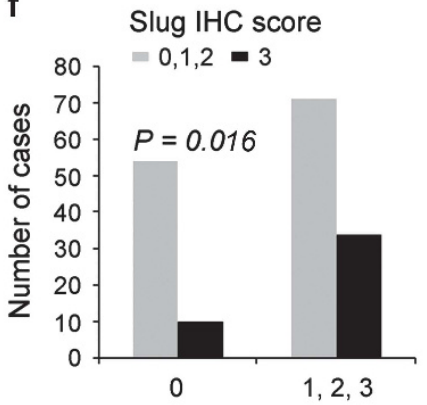

Pellino $1 \mathrm{IHC}$ score g

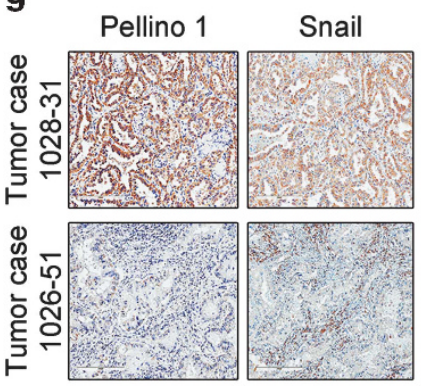

h

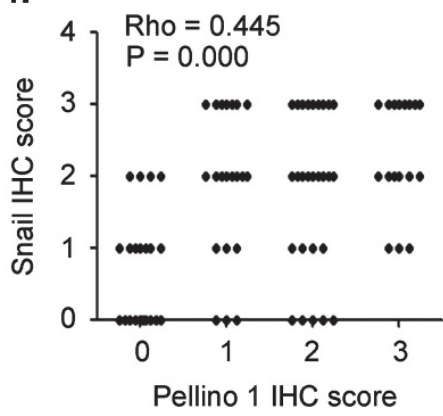

i

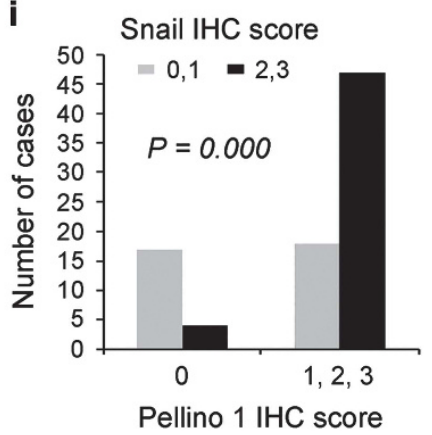

Figure 6 Slug and Snail are required for Pellino-1-mediated increased migration in A549 cells. Pellino-1 expression is correlated with Slug and Snail expression levels in human lung adenocarcinoma tissues. (a and b) A549 cells were transfected with Myc-Pellino-1 in combination with shLuc, shSlug\#1, shSlug\#2 (a) or shSnail\#1, shSnail\#2 (b). At $36 \mathrm{~h}$ post-transfection, the cells were harvested and subjected to immunoblotting for Myc, Slug, Snail, E-cadherin, Vimentin and actin. (c and d) A549 cells were transfected with Myc-Pellino-1 in combination with shLuc, shSlug\#1, shSlug\#2 (c) or shSnail\#1, shSnail\#2 (d) and subsequently subjected to transwell migration assay. Representative images are shown. The scale bars represent $100 \mu \mathrm{m}$. The data are presented as the means \pm S.D. of at least three independent experiments. The $P$-values shown were calculated using unpaired Student's t-test. ${ }^{*} P<0.05 ;{ }^{* *} P<0.01 ;{ }^{* *} P<0.005$. (e) Representative IHC images for Pellino-1 and Slug expression in lung adenocarcinoma patients who showed strong expression for both Pellino-1 and Slug (top) versus those showing no expression for both proteins (bottom). (f) The patients ( $n=169)$ were classified into two groups according to the following Pellino-1 and Slug expression levels: for Pellino-1, those with score 0 versus score 1-3, and for Slug, those with score 0-2 versus score 3. Patients with Pellino-1 expression showed higher Slug expression with statistical significance ( $P=0.016$ by Pearson $\chi^{2}$ test). (g) Representative IHC images for Pellino-1 and Snail expression from lung adenocarcinoma patients who showed strong expression for both Pellino-1 and Snail (left) versus those showing little expression for both proteins (right) are shown. (h) A significantly strong positive correlation between the Pellino-1 and Snail IHC scores was observed (Spearman's $R h o=0.445, P=0.000)$. (i) Patients ( $n=86$ ) were classified into two groups according to the following Pellino-1 and Snail expression levels: for Pellino-1, those with score 0 versus score 1-3, and for Snail, those with score $0-1$ versus score 2-3. Patients with Pellino-1 expression showed significantly higher Slug expression $\left(P=0.000\right.$ by Pearson $\chi^{2}$ test $)$

fusion plasmids. Small hairpin RNAs (shRNAs) targeting human Pellino-1 (shPellino-1), 3'-UTR Pellino-1 (3'-UTR shPellino-1), Slug (shSlug\#1 and shSlug\#2), Snail (shSnail\#1 and shSnail\#2) and the Luciferase (shLuc, as a control) were synthesis using the pSuper vector (Oligoengine, Seattle, WA, USA) according to gene-specific sequences described in Supplementary Materials and Methods. The pcDNA vector encoding Flag-tagged human GSK3 $\beta$ (Flag-GSK3 $\beta$ ), Myc-tagged
Pellino-2 (Myc-Pellino-2) and Myc-tagged Pellino-3 ( $\alpha$ and $\beta$; Myc-Pellino-3 $\alpha$ and Myc-Pellino-3 $\beta$ ) was PCR amplified using oligodT primers. The pcDNA vector encoding Flag-tagged human Slug (Flag-Slug) and the pGEX-KG vector encoding GST-tagged human Slug (GST-Slug) were kindly gifted from Hong-Duck Um (Korea Institute of Radiological \& Medical Sciences, Seoul, Korea). The pcDNA vector encoding Flag-tagged human Snail (Flag-Snail) and the pGEX-KG vector encoding 
GST-tagged human Slug (GST-Snail) were kindly gifted from Bum Joon Park (Pusan National University, Busan, Korea). HA-tagged ubiquitin K48 (HA-Ub K48), HA-tagged ubiquitin K63 (HA-Ub K63), the pEGFP-N3 vector encoding TAP (Strep-Flag-tagged) and His-tagged ubiquitin (His-Ub) were kindly gifted from Hong Tae Kim (Sungkyunkwan University, Suwon, Korea). For transient transfection, the cells were electroporated using a microporator (Digital Biotechnology, Seoul, Korea).

Antibodies and reagents. The following antibodies were used: anti-Pellino-1 (F-7), anti- $\beta$ - TrCP $(\mathrm{H}-85)$ (Santa Cruz Biotechnology, Santa Cruz, CA, USA), antiactin, anti-Flag M2 (Sigma, St. Louis, MO, USA), anti-Slug, anti-Snail, anti-Ecadherin, anti- $\beta$-catenin, anti-Vimentin, anti-ERK1/2, anti-p-ERK1/2 (T202/Y204), anti-Akt, anti-p-Akt (S473), anti-GSK3 $\beta$, anti-p-GSK3 $\beta$ (S9) (Cell Signaling Technology, Danvers, MA, USA), anti-HA, anti-Myc (Roche, Basel, Switzerland). The following reagents were used: LY294002, PD98059 (Calbiochem, San Diego, CA, USA), MG132, cycloheximide, dimethyl sulfoxide (DMSO) (AG Scientific, San Diego, CA, USA) and 3-(4,5-dimethylthiazol-2-yl)-2,5-diphenyltetrazolium bromide (MTT) (Sigma), EGF, mitomycin C (Sigma) and EGFR kinase inhibitor AG1478 (Selleckchem, Houston, TX).

Cell proliferation, transwell migration and invasion assays, and colony-forming assay. Cell proliferation assay and colony-forming assay were performed as described in Supplementary Materials and Methods. Transwell migration assay was performed using uncoated cell culture inserts with $8 \mu \mathrm{m}$ pores (Corning incorporated, Tewksbury, MA, USA) and invasion assay was performed using cell invasion assay kits (Chemicon, Temecula, CA, USA), as described in Supplementary Materials and Methods.

Western blot analysis. The cells were harvested and lysed in nuclear extraction (NE) buffer (20 mM HEPES (pH 7.6), $20 \%$ glycerol, $250 \mathrm{mM} \mathrm{NaCl}$, $1.5 \mathrm{mM} \mathrm{MgCl}_{2}, 0.1 \%$ Triton X-100, $1 \mathrm{mM}$ PMSF, $1 \mathrm{mM}$ DTT and protease inhibitor cocktail (Roche)). Equal amounts of protein were separated through SDS-PAGE and analyzed through immunoblotting with the indicated antibodies.

In vitro binding and immunoprecipitation assays. For the S-tag pulldown assay, 293T cells were adapted to suspension conditions and lysed in NETN buffer (100 mM NaCl, $1 \mathrm{mM}$ EDTA, $20 \mathrm{mM}$ Tris- $\mathrm{HCl}$ (pH 8.0), 0.5\% NP-40) containing $1 \mathrm{mM}$ PMSF, $1 \mathrm{mM}$ DTT and protease inhibitor cocktail. The supernatants were incubated with streptavidin-sepharose beads (Amersham Biosciences, Piscataway, $\mathrm{NJ}, \mathrm{USA}$ ) for $8 \mathrm{~h}$ at $4^{\circ} \mathrm{C}$ and the bound proteins were analyzed via immunoblotting. For the GST pull-down assay, the fusion proteins were adsorbed onto glutathione-protein A/G Sepharose beads (Amersham Biosciences) and incubated with whole cellular extracts from A549 cells or purified His-tagged Pellino1 proteins. The bound proteins were analyzed via immunoblotting. For immunoprecipitation, A549 cell pellets were resuspended in NETN buffer, incubated at $4{ }^{\circ} \mathrm{C}$ for $30 \mathrm{~min}$ and then lysed. The cell lysates were cleared by centrifugation and incubated with an anti-Pellino-1 antibody or normal IgG (control) and then with protein $A / G$ agarose beads, which were later pelleted and analyzed by immunoblotting.

In vivo ubiquitination assays. HCT116 cells were transfected with Myc, Myc-tagged Pellino-1-FL or Pellino-1- $\Delta$ C, Flag-Slug or Flag-Snail, and HA-tagged ubiquitin (HA-Ub), HA-Ub K48 or HA-Ub K63 plasmid in combination. At $48 \mathrm{~h}$ posttransfection, the cells were harvested and cell lysate were collected into two aliquots. One aliquot $(10 \%)$ was used for conventional immunoblotting. The other aliquot $(90 \%)$ were used for the immunoprecipitation with anti-Flag antibody and analyzed by immunoblotting.

In vivo xenograft assay. To evaluate the effect of Pellino-1 on tumor growth, in vivo xenograft tumor model was used. Briefly, 6- to 8-week-old athymic nude (nul nu) mice were housed in laminar-flow cabinets under specific pathogen-free conditions. A549 cells stably expressing shLuc, shPellino-1 or 3'-UTR shPellino-1 $\left(5 \times 10^{6}\right.$ cells) were injected subcutaneously into the flank of mice $(n=5$ mice per group). Tumor size was measured every 3 days using caliper. Tumor volume in $\mathrm{mm}^{3}$ was calculated from the major (a) and minor $(b)$ axis of the tumors using the following formula: $V=a \times b^{2} / 2$. The animal studies and all procedures were approved by the Sungkyunkwan University School of Medicine Institutional Animal Care and Use Committee (IACUC).
Immunohistochemistry for human lung cancer tissues. Formalinfixed paraffin-embedded tumor tissues from patients with pulmonary adenocarcinoma ( $n=491)$, squamous cell carcinoma $(n=59)$ and NSCLC of other histology $(n=26)$ were collected. A tissue microarray with a 2-mm diameter was subjected to IHC. Clinicopathological data were retrieved from the medical records. This study was approved through the Institutional Review Board of Seoul National University Hospital (H-1211-049-440). IHC was performed using the Benchmark XT autostainer (Ventana Medical Systems, Tucson, AZ, USA) with antibodies against Pellino-1 (F-7) (Santa Cruz Biotechnology), Snail (LifeSpan BioSciences, Seattle, WA, USA), and Slug (Abcam, Cambridge, UK). The expression patterns were evaluated based on the intensity and proportion of staining in tumor cells and scored as follows: 0 , negative or staining in $<10 \%$ of tumor cells; 1 , weak; 2 , moderate; or 3 , strong intensity in $>10 \%$ of tumor cells. Statistical analyses were performed using SPSS software (version 21; IBM Corp., New York, NY, USA). Comparisons between variables were performed using the $\chi^{2}$ test or Spearman's correlation test, and two-sided $P$-values $<0.05$ were considered statistically significant.

Quantitative real-time polymerase chain reaction (qRT-PCR). Total RNAs were extracted from cells using RNeasy RNA extraction Mini kit (Qiagen Sciences, Germantown, MD, USA). The reverse transcription was carried out with an EasyScript ${ }^{T M}$ CDNA Synthesis Kit (Applied Biological Materials Inc., Richmond, Canada) using Oligo (dT) primers. For qRT-PCR analyses, a Rotor-Gene $Q$ realtime PCR detection system and SYBR ${ }^{\circledR}$ Green PPCR Master mixes (Qiagen Sciences) were used as follows: $95^{\circ} \mathrm{C}$ for $5 \mathrm{~min}$ and $30 \mathrm{~s}$, and 40 cycles ( $15 \mathrm{~s}$ at $95^{\circ} \mathrm{C}, 1 \mathrm{~min}$ at $60^{\circ} \mathrm{C}$ ). The data were analyzed with a normalized gene expression method (ddCt) using Rotor-Gene $Q$ software (Qiagen Sciences) and the $\beta$-actin were used as a reference for normalization. qRT-PCR was performed according to gene-specific sequences described in Supplementary Materials and Methods.

\section{Conflict of Interest}

The authors declare no conflict of interest.

Acknowledgements. This study was financially supported through a National Research Foundation grant from the Korean government (MEST; 2013R1A1A2063952 and 2012M3A9A8053102) and a grant of the Korea Health Technology R\&D Project through the Korea Health Industry Development Institute (KHIDI), funded by the Ministry of Health \& Welfare, Republic of Korea (H114C0069). We would like to thank Dr. Hong-Duck Um (Korea Institute of Radiological \& Medical Sciences), Dr. Bum Joon Park (Pusan National University) and Dr. Hong Tae Kim (Sungkyunkwan University) for providing plasmids.

\section{Author contributions}

$\mathrm{G}-\mathrm{HH}$ and $\mathrm{YKJ}$ designed the study, developed and performed the experiments, analyzed the data, and wrote the manuscript. CKK, KRH, H-YP and JK performed the experiments and analyzed the data. DHC and C-WL designed the study, analyzed the data, supervised the study and wrote the manuscript.

1. Nieto MA. The ins and outs of the epithelial to mesenchymal transition in health and disease. Annu Rev Cell Dev Biol 2011; 27: 347-376.

2. De Craene B, Berx G. Regulatory networks defining EMT during cancer initiation and progression. Nat Rev Cancer 2013; 13: 97-110.

3. Gao D, Vahdat LT, Wong S, Chang JC, Mittal V. Microenvironmental regulation of epithelialmesenchymal transitions in cancer. Cancer Res 2012; 72: 4883-4889.

4. Thiery JP, Acloque $\mathrm{H}$, Huang RY, Nieto MA. Epithelial-mesenchymal transitions in development and disease. Cell 2009; 139: 871-890.

5. Polyak K, Weinberg RA. Transitions between epithelial and mesenchymal states: acquisition of malignant and stem cell traits. Nat Rev Cancer 2009; 9: 265-273.

6. Cano A, Perez-Moreno MA, Rodrigo I, Locascio A, Blanco MJ, del Barrio MG et al. The transcription factor snail controls epithelial-mesenchymal transitions by repressing E-cadherin expression. Nat Cell Biol 2000; 2: 76-83.

7. Graham TR, Zhau HE, Odero-Marah VA, Osunkoya AO, Kimbro KS, Tighiouart M et al. Insulin-like growth factor-l-dependent up-regulation of ZEB1 drives epithelial-tomesenchymal transition in human prostate cancer cells. Cancer Res 2008; 68: 2479-2488.

8. Vandewalle C, Comijn J, De Craene B, Vermassen P, Bruyneel E, Andersen $\mathrm{H}$ et al. SIP1/ ZEB2 induces EMT by repressing genes of different epithelial cell-cell junctions. Nucleic Acids Res 2005; 33: 6566-6578. 
9. Rosivatz E, Becker I, Specht K, Fricke E, Luber B, Busch R et al. Differential expression of the epithelial-mesenchymal transition regulators snail, SIP1, and twist in gastric cancer. $A m$ J Pathol 2002; 161: 1881-1891.

10. Willis BC, Borok Z. TGF-beta-induced EMT: mechanisms and implications for fibrotic lung disease. Am J Physiol Lung Cell Mol Physiol 2007; 293: L525-L534.

11. Larue L, Bellacosa A. Epithelial-mesenchymal transition in development and cancer: role of phosphatidylinositol 3' kinase/AKT pathways. Oncogene 2005; 24: 7443-7454.

12. Turley EA, Veiseh M, Radisky DC, Bissell MJ. Mechanisms of disease: epithelialmesenchymal transition-does cellular plasticity fuel neoplastic progression? Nat Clin Pract Oncol 2008; 5: 280-290.

13. Jung KW, Won YJ, Kong HJ, Oh CM, Cho H, Lee DH et al. Cancer statistics in Korea: incidence, mortality, survival, and prevalence in 2012. Cancer Res Treat 2015; 47: 127-141.

14. Siegel RL, Miller KD, Jemal A. Cancer statistics, 2015. CA Cancer J Clin 2015; 65: 5-29.

15. Feng Q, Hawes SE, Stern JE, Wiens L, Lu H, Dong ZM et al. DNA methylation in tumor and matched normal tissues from non-small cell lung cancer patients. Cancer Epidemiol Biomarkers Prevent 2008; 17: 645-654.

16. Nakata S, Sugio K, Uramoto H, Oyama T, Hanagiri T, Morita M et al. The methylation status and protein expression of $\mathrm{CDH1}, \mathrm{p} 16(\mathrm{INK} 4 \mathrm{~A})$, and fragile histidine triad in nonsmall cell lung carcinoma: epigenetic silencing, clinical features, and prognostic significance. Cancer 2006; 106: $2190-2199$

17. Shih JY, Yang PC. The EMT regulator slug and lung carcinogenesis. Carcinogenesis 2011; 32: 1299-1304.

18. Sato M, Shames DS, Hasegawa Y. Emerging evidence of epithelial-to-mesenchyma transition in lung carcinogenesis. Respirology 2012; 17: 1048-1059.

19. Hui L, Zhang S, Dong X, Tian D, Cui Z, Qiu X. Prognostic significance of twist and N-cadherin expression in NSCLC. PLoS One 2013; 8: e62171.

20. Miura N, Yano T, Shoji F, Kawano D, Takenaka T, Ito K et al. Clinicopathological significance of Sip1-associated epithelial mesenchymal transition in non-small cell lung cancer progression. Anticancer Res 2009; 29: 4099-4106.

21. Rich T, Allen RL, Lucas AM, Stewart A, Trowsdale J. Pellino-related sequences from Caenorhabditis elegans and Homo sapiens. Immunogenetics 2000; 52: 145-149.

22. Butler MP, Hanly JA, Moynagh PN. Kinase-active interleukin-1 receptor-associated kinases promote polyubiquitination and degradation of the Pellino family: direct evidence for PELLINO proteins being ubiquitin-protein isopeptide ligases. J Biol Chem 2007; 282 29729-29737.

23. Ordureau A, Smith H, Windheim M, Peggie M, Carrick E, Morrice N et al. The IRAKcatalysed activation of the E3 ligase function of Pellino isoforms induces the Lys63-linked polyubiquitination of IRAK1. Biochem J 2008; 409: 43-52.

24. Moynagh PN. The roles of Pellino E3 ubiquitin ligases in immunity. Nat Rev Immunol 2014; 14: 122-131.

25. Chang M, Jin W, Chang JH, Xiao Y, Brittain GC, Yu J et al. The ubiquitin ligase Peli1 negatively regulates $T$ cell activation and prevents autoimmunity. Nat Immunol 2011; 12: 1002-1009.

26. Chang M, Jin W, Sun SC. Peli1 facilitates TRIF-dependent Toll-like receptor signaling and proinflammatory cytokine production. Nat Immunol 2009; 10: 1089-1095.

27. Choi KC, Lee YS, Lim S, Choi HK, Lee CH, Lee EK et al. Smad6 negatively regulates interleukin 1-receptor-Toll-like receptor signaling through direct interaction with the adaptor Pellino-1. Nat Immunol 2006; 7: 1057-1065.

28. Schauvliege R, Janssens S, Beyaert R. Pellino proteins: novel players in TLR and IL-1 R signalling. J Cell Mol Med 2007; 11: 453-461.

29. Jiang Z, Johnson HJ, Nie H, Qin J, Bird TA, Li X. Pellino 1 is required for interleukin-1 (IL-1)mediated signaling through its interaction with the IL-1 receptor-associated kinase 4 (IRAK4)-IRAK-tumor necrosis factor receptor-associated factor 6 (TRAF6) complex. J Biol Chem 2003; 278: 10952-10956.

30. Park HY, Go H, Song HR, Kim S, Ha GH, Jeon YK et al. Pellino 1 promotes lymphomagenesis by deregulating BCL6 polyubiquitination. J Clin Invest 2014; 124: 4976-4988.

31. Batlle E, Sancho E, Franci C, Dominguez D, Monfar M, Baulida J et al. The transcription factor snail is a repressor of $\mathrm{E}$-cadherin gene expression in epithelial tumour cells. Nat Cell Biol 2000; 2: 84-89.

32. Bolos V, Peinado H, Perez-Moreno MA, Fraga MF, Esteller M, Cano A. The transcription factor Slug represses E-cadherin expression and induces epithelial to mesenchyma transitions: a comparison with Snail and E47 repressors. J Cell Sci 2003; 116(Pt 3): 499-511.

33. Hajra KM, Chen DY, Fearon ER. The SLUG zinc-finger protein represses E-cadherin in breast cancer. Cancer Res 2002; 62: 1613-1618.

34. Boyer B, Valles AM, Edme N. Induction and regulation of epithelial-mesenchymal transitions. Biochem Pharmacol 2000; 60: 1091-1099.
35. Zhou BP, Deng J, Xia W, Xu J, Li YM, Gunduz M et al. Dual regulation of Snail by GSK-3beta-mediated phosphorylation in control of epithelial-mesenchymal transition. Nat Cell Biol 2004; 6: 931-940.

36. Peinado $H$, Quintanilla M, Cano A. Transforming growth factor beta-1 induces snail transcription factor in epithelial cell lines: mechanisms for epithelial mesenchymal transitions. J Biol Chem 2003; 278: 21113-21123.

37. Kao SH, Wang WL, Chen CY, Chang YL, Wu YY, Wang YT et al. GSK3beta controls epithelial-mesenchymal transition and tumor metastasis by CHIP-mediated degradation of Slug. Oncogene 2014; 33: 3172-3182.

38. Yamauchi M, Yamaguchi R, Nakata A, Kohno T, Nagasaki M, Shimamura T et al. Epidermal growth factor receptor tyrosine kinase defines critical prognostic genes of stage I lung adenocarcinoma. PLoS One 2012; 7: e43923.

39. Chang YS, Wang L, Liu D, Mao L, Hong WK, Khuri FR et al. Correlation between insulin-like growth factor-binding protein-3 promoter methylation and prognosis of patients with stage I non-small cell lung cancer. Clin Cancer Res 2002; 8: 3669-3675.

40. Piyathilake CJ, Frost AR, Manne U, Weiss H, Bell WC, Heimburger DC et al. Differential expression of growth factors in squamous cell carcinoma and precancerous lesions of the lung. Clin Cancer Res 2002; 8: 734-744.

41. Meek CL, Wallace AM, Forrest LM, McMillan DC. The relationship between the insulin-like growth factor-1 axis, weight loss, an inflammation-based score and survival in patients with inoperable non-small cell lung cancer. Clin Nutr 2010; 29: 206-209.

42. Hasegawa Y, Takanashi S, Kanehira Y, Tsushima T, Imai T, Okumura K. Transforming growth factor-beta1 level correlates with angiogenesis, tumor progression, and prognosis in patients with nonsmall cell lung carcinoma. Cancer 2001; 91: 964-971.

43. Moreno-Bueno G, Cubillo E, Sarrio D, Peinado H, Rodriguez-Pinilla SM, Villa S et al. Genetic profiling of epithelial cells expressing E-cadherin repressors reveals a distinct role for Snail, Slug, and E47 factors in epithelial-mesenchymal transition. Cancer Res 2006; 66: 9543-9556.

44. Moynagh PN. The Pellino family: IRAK E3 ligases with emerging roles in innate immune signalling. Trends Immunol 2009; 30: 33-42.

45. Huoh YS, Ferguson KM. The pellino e3 ubiquitin ligases recognize specific phosphothreonine motifs and have distinct substrate specificities. Biochemistry 2014; 53: 4946-4955.

46. Merikallio H, T TT, Paakko P, Makitaro R, Kaarteenaho R, Lehtonen S et al. Slug is associated with poor survival in squamous cell carcinoma of the lung. Int $\mathrm{J}$ Clin Exp Pathol 2014; 7: 5846-5854.

47. Ono M, Hirata A, Kometani T, Miyagawa M, Ueda S, Kinoshita $\mathrm{H}$ et al. Sensitivity to gefitinib (Iressa, ZD1839) in non-small cell lung cancer cell lines correlates with dependence on the epidermal growth factor (EGF) receptor/extracellular signal-regulated kinase 1/2 and EGF receptor/Akt pathway for proliferation. Mol Cancer Ther 2004; 3: 465-472.

48. Dan HC, Cooper MJ, Cogswell PC, Duncan JA, Ting JP, Baldwin AS. Akt-dependent regulation of NF-\{kappa\}B is controlled by mTOR and Raptor in association with IKK. Genes Dev 2008; 22: 1490-1500.

49. Meng F, Liu L, Chin PC, D'Mello SR. Akt is a downstream target of NF-kappa B. J Biol Chem 2002; 277: 29674-29680.

50. Kumar M, Allison DF, Baranova NN, Wamsley JJ, Katz AJ, Bekiranov S et al. NF-kappaB regulates mesenchymal transition for the induction of non-small cell lung cancer initiating cells. PLoS One 2013; 8: e68597.

51. Grille SJ, Bellacosa A, Upson J, Klein-Szanto AJ, van Roy F, Lee-Kwon W et al. The protein kinase Akt induces epithelial mesenchymal transition and promotes enhanced motility and invasiveness of squamous cell carcinoma lines. Cancer Res 2003; 63: 2172-2178.

52. Chou TY, Chen WC, Lee AC, Hung SM, Shih NY, Chen MY. Clusterin silencing in human lung adenocarcinoma cells induces a mesenchymal-to-epithelial transition through modulating the ERK/Slug pathway. Cell Signal 2009; 21: 704-711.

(1) This work is licensed under a Creative Commons cc) Attribution 4.0 International License. The images or other third party material in this article are included in the article's Creative Commons license, unless indicated otherwise in the credit line; if the material is not included under the Creative Commons license, users will need to obtain permission from the license holder to reproduce the material. To view a copy of this license, visit http:// creativecommons.org/licenses/by/4.0/

(C) The Author(s) 2017 